\title{
SURINAAMSCHE ONDERWERPEN OP HET AMERIKANISTEN-CONGRES TE HAMBURG
}

\author{
DOOR
}

\author{
JHR. L. C. VAN PANHUYS
}

(Vervolg en slot)

\section{Boschnegerornamenten}

Die Buschneger in Surinam ${ }^{1}$ ) besitzen einen reichen Schatz von noch sehr wenig bekannten Ornamenten, über deren Bedeutung gleichfalls bisher noch wenig veröffentlicht wurde. Ihre Tätowierfiguren sind wohl am ersten der Gefahr ausgesetzt zu verschwinden, da die Buschneger, sobald sie zum Christentum bekehrt worden sind, das Tätowieren aufgeben.

In uneigennütziger Weise haben die Herren C. H. de Goeje und $\mathrm{H}$. Bisschop van Tuinen die Güte gehabt während der fünften Surinam-Expedition (Tumak-Humak, 1907), neben allen andern umfangreichen Arbeiten, 21 Abbildungen (Skizzen) von Tätowier-figuren zu machen, und von mehrer derselben die Erklärung und Bedeutungen erfahren, wofür Verfasser dieses 1909 den ebengenannten gewissenhaften Beobachtern seinen besten Dank für ihre tüchtigen Leistungen ganz ergebenst ausgesprochen hat ${ }^{2}$ ). Aber auch leutnant zur See R. H. Wymans hat sich während einer folgenden Expedition (Surinam-Fluss) die Mühe gegeben u. a. Besonderheiten über die Tätowierung bei den SaramakkanerBuschnegern aufzuzeichnen ${ }^{3}$ ). Seine Notizen sind, obwohl kurz,

$\left.{ }^{1}\right)$ Wir bitten um ein sprachhistorische Untersuchung des Namens „Surinam”, c.q. eine Rechtschreibung und Verbesserung. Wie kam man dazu, den, doch saüber indianischen, Namen dergestalt und nicht: „Suriname” zu schreiben? Sieht „Quelques chansons et quelques danses", usw., Notiz 1. blz. 211 W. I. G. Man schreibt und sagt doch auch: Coppename-Expedition, und niemals: Coppenam-Expedition.

2) Verhandlungen des XVI Intern. Amerikanisten-Kongresses, Wien 1909, S. 527. Artikel Tätowieren, mit 3 Figuren (14 Formen).

s) S. 685 u. 686 Tijdschrift Kon. Ned. Aardrijkskundig Genootschap, 1910 
doch wertvoll. Melden wir noch, dasz Major Bakhuis fünf deutliche Zeichnungen von Tätowierfiguren gegeben hat mit einer kurzen Bemerkung ${ }^{1}$ ), und dasz ein kurze Notiz von meiner Hand vorkommt auf S. 437-439 der Verhandlungen des XIV Amerikanisten-Kongresses, Stuttgart 1906, so haben wir, glaube ich, ungefähr alles genannt was über die Tätowierung bei Buschnegern veröffentlicht worden ist, - von dem Wenigen - nur einige Zeilen-, was Kappler und Crevaux davon gesagt haben abgesehen (in unserer Notiz angedeutet). Doch wohl müssen wir hervorheben was Joest in seinem „Ethnographisches und Verwandtes", Int. Archiv für Ethnographie, Suppl. zu Band V, 1893, S. 49 über das Tätowieren schreibt, weil er es so hübsch verstanden hat uns die Tätowiersitte zu schildern in der Umgebung wo der Brauch lebt. Das Auffällendste an dem Äuszern der Buschneger beider Geschlechter, ist ihre Tätowierung, sagt er so ungefähr, bzw. die über den ganzen Körper sich erstreckende „Verschönerung" der Haut durch Ziernarben. Selten fehlen die afrikanischen Wangen- und Schläfenschmisse.

Vielfach ist der ganze Körper mit wirklich zierenden und zierlichen kleinen Narben, die zu sehr gefälligen Mustern zusammengestellt werden, bedeckt. Die Narben werden in der bekannten Weise dadurch hergestellt, dasz man eine kleine Hautfalte zuzammenkneift und deren Scheitel durch einen ziemlich tiefen, etwa $1 \mathrm{~cm}$. langen, Schnitt aufritzt. Hierzu dient in Surinam meist ein Rasiermesser (Wymans 1.c. gibt uns den Namen: simin-néfi, néfi $=\mathrm{E}$. Knife, Alt H. Knijf, und sagt, dasz meistenteils Weiber die Operation ausführen). Bakhuis l.c. sagt n.u. M. richtig: „Het vel wordt telkens tusschen 2 vingers genomen en dan 2 sneetjes van 5 à $6 \mathrm{~mm}$. naast elkander daarin gemaakt" ${ }^{2}$ ).

Sehr richtig urteilt Joest, dasz ein mit Narben verzierter Rücken, oder zumal bei den jungen Mädchen, die ornamentierten Busen und Oberschenkel, indessen wirklich entschieden hübscher aussehen, als die entsprechenden Teile der gewöhnlichen Neger. Und ein Schalk kommt wohl dazu und behauptet dasz die Mädchen

1) Bericht der Coppename-Expedition, Leiden, 1902 Ueberdruck Tijdschrift Kon. Ned. Aardrijkskundig Genootschap, 1902, S. 35 und Abbildung gegenüber S. 148 .

$\left.{ }^{2}\right)$ Joest spricht auch vom Punktieren der Haut und sagt: Einreiben der betreffenden Stellen mit Rusz kommt vielfach zur Anwendung. Wymans 1.c. nennt eine andere Einreibung. Ich selbst habe nur von einer Einschneidung und nicht von Punktierung gehört, und auch nicht von Anwendung von Russ, sondern nur von Holzkohle. In meinen Notizen steht: Holzkohle verursacht die Aufschwellung. 
ihre Narben gern und ohne Ziererei zeigen, so wie eine Europäerin ihre Schmucksachen: dabei lassen sie in ihrer Harmlosigkeit allerdings zuweilen gewisse narbengezierte Stellen ihres schönen Körpers bewundern, die man bei uns zu den diskretesten zu rechnen pflegt.

Ein Beobachter hat mir seiner Zeit geschrieben, dasz Frauen die meisten ,kotti-kotti” (Tätowierungen, buchstäblich: Einschnitte) haben in der Umgebung der Geschlechtsteile, also verborgen unter dem Hüftentuch, weil sie beim Koitus eine angenehme Reibung verursachen oder aber, wenn die Figuren (ngaté genannt) auf den Rücken geschnitten worden sind, um die Körperteile an dieser Stelle beim Beischlaf zu reiben.

Wir müssen also bei der Erklärung der Tätowierfiguren darauf bedacht sein, dasz deren Zweck und Bedeutung mit dem Liebesleben verbunden können sein und werden in diesem Gedanken bestärkt wenn wir lesen was Professor und Frau Melville J. Herskovits, von Cornell-University, Evanston, Illinois, Vereinigte Staaten von N. Amerika, uns mitteilen in ihrem höchst interessanten Artikel: „Bush-Negro Art” 1), The Arts New York, The Arts Publishing Corporation, October 1930 nämlich, dasz einige der Tätowierungen in Umrissen und Symbolik mit den Holzschnitt Ornamenten übereinstimmen; und dasz jene Ornamente eine Geschlechts-Symbolik enthalten die ihre Kunst sehr beeinfluszt.

Schlangen- und Menschenfiguren sind haüfig mit kupfernen Nägeln verziert, die Tätowier-Figuren auf Kopf- und Gesicht, oder auf dem Leib vorstellen. Der Gebrauch von kupfernen Nägeln auf dem Leib einer Schlange, so sagt der Artikel Bush-Negro Art, ist merkwürdig, weil es klar ist, dasz die Absicht war der Schlange menschliche Eigenschaften zuzulegen.

Ich denke, man wird nicht so leicht die Erklärung aller Tätowier-ornamente bekommen. Begreifen der immer stark stilisierten Buschnegerkunst ist unmöglich ohne Hilfe der Buschneger, und sie verstehen, so sagen Herr Dr. und Frau Herskovits sehr richtig, ausgezeichnet die Kunst Fragen nach der Bedeutung von Verzierungen auszuweichen. „The fact is that we were entirely unsuccesful in obtaining a single explanation of design during our first visit" (nämlich: während der erste Reise im Jahre 1928), bemerken Herr und Frau Herskovits dazu. - Nehmen wir ein Beispiel. Man sieht eine Tätowierfigur auf dem Nac-

1) Zie het artikel „Boschnegerkunst” in het September-nummer van de West-Indische Gids. 
ken eines Buschnegers und fragt nach dem Namen. Jetzt kann er antworten: neki-kokotti (Nacken-tätowierung); daran hat man nicht viel, man musst aber darauf achtgeben, ob vielleicht jene Tätowierung immer, oder oft, auf dem Nacken vorkommt. Wenn man Glück hat, erfährt man auch den eigentlichen Namen, sagen wir z.B.: Ananassi (Ananas, Frücht oder Pflanze). Aber jetzt müssen wir noch wissen: Warum haben Sie da eine Ananas, m.a.W., mit welcher symbolischen Bedeutung ist eine Ananas da angebracht worden? Und das erfährt man eben nicht.

Nur durch Vergleichung vieler Figuren, und Erklärungen von zuverlässigen Buschnegern, Männern und Frauen, wird man sein Ziel erreichen.

Er volgt nu in het Duitsche manuscript een uitvoerige beschrijving van een en twintig afbeeldingen van tatoeage-ornamenten, met de afbeeldingen zelf, welke in dit uittreksel niet worden opgenomen. Wie dit onderdeel van de boschnegerornamentiek wil bestudeeren, zal afbeeldingen en beschrijving kunnen aantreffen in de „Verhandlungen” van het 24ste Amerikanisten-Congres. In de desbetreffende beschrijving wordt er o.a. de aandacht op gevestigd, dat een slangenvormige korjaalversiering, welke door den heer Bakhuis in zijn aangehaald „Bericht” tegenover blz. 148 is afgebeeld, waarschijnlijk dezelfde beteekenis heeft als het door den heer en mevrouw Herskovits in hun artikel „Bush Negro Art” beschreven en afgebeelde ornament, dat de boschneger „man ko muje", man- en vrouw-figuur noemt, hetwelk op zóódanige wijze is gestiliseerd dat, zooals het echtpaar Herskovits opmerkt: ,only the limbs are shown". Van een andere afbeelding zeggen dr. en mevr. H.: „Although so much richer in detail, the symbolism is relatively the same" en zij voegen daaraan toe, dat beschaving en kunst van de boschnegers een zekere eenheid vertoonen. Een van de tatoeëer-figuren van de verzameling de G. en B. v. T. is een aardige voorstelling van een mij onbekend insekt, kirifaja (vuurof lichtblusscher); een ander komt overeen met een door Wymans vermeld patroon, dat man en vrouw wederkeerig bij elkander op den rug in de taille aanbrengen, indien zij bijzonder veel van elkaar houden.

Verder wordt de beteekenis verklaard van door den heer de G. nageteekende ornamenten, die djoeka-vrouwen in kleine kalebassen hebben ingesneden, n.l. van een gestiliseerden vogel, een vleermuis en een vogel-figuur. Ook van een ornament in de neb van een korjaal, een man in een doodkist voorstellend. 


\section{4}

Zeer merkwaardig zijn de afwrijvingen met zwarte was en Japansch papier door de heeren de G. en B. v. T. van boschnegerornamenten, genomen volgens de methode van Hjalmar Stolpe, zooals b.v. die van een waschgoedklopper, die vermoedelijk een vodoe-god voorstelt met een zeer groot geslachtsdeel. Op een sierparel schijnt een man door een schorpioen (totem- of familieteeken?) te zijn voorgesteld. Bij een vrouw-figuur ziet men een ronde symbolische vrucht uit de schoot (baarmoeder) te voorschijn komen. Bij een andere afbeelding van eene vrouw kan men, met behulp van de door dr. Herskovits gegevene verklaringen, gevoegd bij die van den maker, den boschneger Wintimatti, opmaken, dat bedoeld wordt weer te geven, dat de vrouw er naar verlangt dat haar echt vruchtbaar zij (twee lijnen gaan, van haar hoofd, naar een maansikkel, het zinnebeeld van vruchtbaarheid boven haar); voorts, dat haar verzekerd wordt, dat haar wensch zal kunnen worden vervuld, indien zij van haar man houdt en hij van haar (twee liefdes-pijlen staan, tusschen haar hoofd en den maansikkel, in tegengestelde richting en kunnen beteekenen: ik zend liefde uit en ik ontvang wederliefde) en eindelijk, dat de paring lang moet duren (zooals b.v. plaats heeft, wanneer de man van een reis thuis komt). Daarop doelt het ornament dat de bosch neger noemt: ,langa tem".

Aan het bovenstaande laten wij thans de volgende slotopmerkingen in de Duitsche taal aansluiten.

Wir können bevor wir ein Schlusswort aussprechen, noch eine kurze Bemerkung über tätowieren hinzufügen. Niemals gibt es einen Tätowierungszwang. Man fängt damit an einem „pikien Ningré" drei kleine Einschnitte zu geben auf die Maus der Hand zwischen Daumen und Zeigefinger (rechte oder linke. Hand). Darauf folgt, das Kinn, und dann die Stirn. Hat ein junger Mann blosz einen Kinneinschnitt (z.B. vier Schnittchen), so darf er noch nicht mit einer Frau (einem Mädchen) Umgang haben; wohl falls er einen Stirneinschnitt hat. Zugleich folgen Einschnitte unter die Augen. Denn diese Einschnitte tun sehr weh, und wenn man mit einer Frau Umgang gehabt hat, muss man diesen Schmerz ertragen können. Nicht: weil die Schnitte gemacht worden sind, sondern weil bei jungen Leuten die Geschlechtsreife eintritt, wird ein Tanz gehalten. Es folgen Schnitte auf Wangen, Bauch, Beine und Rücken (von unten nach oben) so bald man es selbst will. 


\section{NACHTRAG}

Am neunten Januar 1931 haben wir im Kolonial Institut in Amsterdam für die Vereine „Oost en West" und „Hou en Trouw” einen Vortrag mit Lichtbildern gehalten, über merkwürdige Beispiele von ursprünglicher Kunst in Surinam und sonstwo ${ }^{1}$ ), und da angeführt, dasz es wünschenswert wäre in Zukunft zur Abfassung einer „Grammar of Ornaments" zu kommen, wie Owen Jones in 1856 eine veröffentlichte, aber dann nicht mit ganz unverstandenen Ornamenten zu kommen, sondern mit Ornamenten und vor allem mit deren Erklärung. Eine Arbeit deren hervorragende Bedeutung sich jeder Ethnologe und Praehistoriker klar vor Augen stellen soll, die aber vielleicht erst nach einer oder mehreren Generationen fertig werden kann.

Die Vorarbeiten könnten anfangen mit einer Zusammenstellung von Ornamente, wie es Prof. Alfred L. Kroeber getan hat in seinem Buch von der Frau Morris K. Jesup-de Witt-Expedition ${ }^{2}$ ), The Arapaho, Bulletin of the American Museum of Natural History, Vol. XVIII, Part. I, pp. 1-150, New York, Sept. 3., 1902, wo auf Seite 138-143 eine kurze Zusammenfassung gegeben wird von nicht weniger als 458 Figuren.

Wir stellen uns vor, dasz jede Nation, die über den nötigen wissenschaftlichen Kräften verfügt um sich der Untersuchung der Volksstämme, die sich durch merkwürdige Ornamentik auszeichnen, widmen zu können es sich zur Ehre rechnen wird, die Ornamente selbst und ins besondere deren Erklärung zu sammeln in solcher Weise, dasz spätere Vergleichung mit den Ornamenten andrer Völker leicht geschehen kann. So könnte vielleicht in viele Staaten so nicht mit der Zeit in jedem Staat, c.q. für jeden Gebietsteil, ein Ornamente-Institut oder eine Ornamente-Arbeitskammer gegründet werden, und irgendwo ein Ornamente-Sammlungs-Institut, wo, aus der ganzen Welt, die Ornamente Untersuchungs-Resultate zusammen gebracht würden. Aber es ist nicht blosz Pionierarbeit zu tun. Schon ist eine ungeheure Menge von Ornamenten mit ihren Erklärungen bekannt, und könnte schon gleich von dem Sammlungs-Institut in eine einfache und vergleichbare Form gebracht werden ${ }^{3}$ ).

\footnotetext{
1) Nämlich über: Holzschnittwerke in Norwegischen hölzernen Kirchen; Praehistorische Symbolik; Ornamente der Buschneger und der Indianern; Ornamente in Brasilien; in Borneo; von Nord-West Indianern in Nord-Amerika; bei den Amur-Stämmen.

${ }^{2}$ ) Mrs. Morris K. Jesup war Holländerin.

s) Wir dürfen hier wohl mit vorzüglicher Hochachtung und Freude
} 
Für die Niederlände liegt dabei die Aufgabe vor, die angegebene Arbeit zu unternehmen mit Bezug auf Niederländisch Ost- und West-Indien. In Surinam soll man sich eifrig bemühen die Ornamenterklärungen der Buschneger (und natürlich so viel wie möglich auch die Ornamente selbst) zu erhalten. Für Niederländisch Ost-Indiën ist uns in dieser Hinsicht nur bekannt, dasz schon vor langen Jahren Prof. A. R. Hein: Die bildenden Künste bei den Dajaks auf Borneo veröffentlicht hat und dasz wertvolle Elemente durch Prof. A. W. Nieuwenhuis bekannt geworden sind. Die kleine aber vorzügliche Arbeit vom Bruder des Herrn Professors, Dr. Wilhelm Hein „Die Menschengestalt in Flechtwerken", Band XXI. Mitt. Anthrop. Gesellschaft, Wien, 1891, wird, unseres Erachtens, als Ausgangspunkt können dienen.

Selbstverständlich soll jeder fremde Gelehrte der sich zur Untersuchungsarbeit in dieser Richtung berufen fühlt, von der betreffenden Regierung Hilfe und wo möglich auch Unterstützung empfangen. Und jetzt noch das wichtigste. Niemals unterlasse es ein Reisender, ein Forscher, himfort das Äuszerste zu tun, um Erklärungen der von ihm gesammelten Ornamente zu finden ${ }^{1}$ ).

über alles was sie uns brachten die Namen nennen von $\mathrm{H}$. Balfour, F. Boas, A. C. Haddon, Al. R. Hein, Wilhelm Hein, A. L. Kroeber, B. Laufer, K. T. Preuss, H. Schurtz, K. von den Steinen und Hjalmar Stolpe.

$\left.{ }^{1}\right)$ Es sei uns gestattet hier die Literatur-Angaben zu ergänzen die wir unseren Artikel beigefügt hatten: Quelques ornements des nègres des bois de la Guyane néerlandaise, Atti del XXII Congresso Internaz. degli Americanisti, S. 231-276, II, nämlich:

Owen Jones, Grammar of Ornament, London 1856.

F. Boas, The decorative Art of the Indians of the North Pacific Coast. (Bulletin American Museum of Natural History, Vol. IX. Art. X, pp. 123-176. New York 1897).

B. Laufer: The decorative Art of the Amur Tribes. Memoirs of the American Museum of Natural History. Vol. VII. Publication of the Jesup North Pacific Expedition 1902.

A. L. Kroeber: The Arapaho. Bulletin American Museum of Natural History. Vol. XVIII. Part. 1, pp. 1-150. New York 1902.

Eric Boman: Antiquités de la région andine de la république argentine. Paris 1908.

G. Lindblom: Einige Details in der Ornamentik der Buschneger Surinams. Riksmuseet Etnografiska Avdelning, Smarre Meddalanden, Stockholm 1926.

Melville J. Herskovits and Frances S. Herskovits: Bush-Negro Art. The Arts, New York 232 East, 54th Street, October 1930. 